Article

\title{
Dissipativity of Fractional Navier-Stokes Equations with Variable Delay
}

\author{
Lin F. Liu ${ }^{1}$ and Juan J. Nieto ${ }^{2, *}$ \\ 1 School of Mathematics, Northwest University, Xi'an 710075, China; liulinfang2020@nwu.edu.cn \\ 2 Instituto de Matemáticas, Universidade de Santiago de Compostela, 15782 Santiago de Compostela, Spain \\ * Correspondence: juanjose.nieto.roig@usc.es
}

Received: 14 October 2020; Accepted: 9 November 2020; Published: 16 November 2020

\begin{abstract}
We use classical Galerkin approximations, the generalized Aubin-Lions Lemma as well as the Bellman-Gronwall Lemma to study the asymptotical behavior of a two-dimensional fractional Navier-Stokes equation with variable delay. By modifying the fractional Halanay inequality and the comparison principle, we investigate the dissipativity of the corresponding system, namely, we obtain the existence of global absorbing set. Besides, some available results are improved in this work. The existence of a global attracting set is still an open problem.
\end{abstract}

Keywords: fractional Navier-Stokes equations; variable delay; modified fractional Halanay inequality; generalized comparison principle; dissipativity

\section{Introduction}

We study the longtime behavior of the following two-dimensional Navier-Stokes equation of fractional order with variable delay on a bounded domain $\Omega \subset \mathbb{R}^{2}$,

$$
\begin{gathered}
D_{t}^{\alpha} u-v \Delta u+(u \cdot \nabla) u+\nabla p=f(t)+g\left(t, u_{t}\right), \text { in }(0, T) \times \Omega, \\
\operatorname{div} u \equiv 0, \text { in }(0, T) \times \Omega, \\
u=0, \text { on }(0, T) \times \partial \Omega, \\
u(t, x)=\phi(t, x), t \in[-h, 0], x \in \Omega,
\end{gathered}
$$

where $D_{t}^{\alpha}$ is a fractional derivative of order $\alpha \in(0,1), T>0, \Omega \subset \mathbb{R}^{2}$ is a bounded open set with regular boundary $\partial \Omega, v>0$ is the kinematic viscosity, $u$ is the velocity field of the fluid, $p$ is the pressure, $\phi$ is the initial datum, $h>0$ is a constant, $f$ is an external force field without delay, and $g$ is the external force containing some functional delay. We will refer to (1)-(4) as problem $(P)$.

In fact, hereditary characteristics are ubiquitous in engineering, biology and physics. For example, feedback control problem, immune systems, soft matter with viscoelasticity [1] could all have hereditary properties (including memory, variable delay or distributed delay, constant delay, etc). The delay term is very often denoted by a function $u_{t}(\cdot)$ defined on some interval $[-h, 0]$ (here $h$ could be $-\infty$ ). The memory effect is modeled by using fractional calculus, which actually has been widely applied in many sciences [2-5]. We would like to mention that the concept of fractional calculus was raised by L'Hospital, who wrote to Leibniz in the year 1695, seeking the meaning of $\frac{d^{n} y}{d x^{n}}$ when $n=\frac{1}{2}$. However, it only became popular in practical applications in the past few decades. Several kinds of definitions of fractional derivatives have 
been introduced [2], but maybe the most commonly used nowadays are the so-called Riemann-Liouville derivative and Caputo derivative. More definitions for Riemann-Liouville and Caputo derivative can be found in $[3,6,7]$.

It is worth pointing out that using a convolution group, $\mathrm{Li}$ and $\mathrm{Liu}$ [8] introduced a generalized definition of Caputo derivative of order $\alpha \in(0,1)$, and built a convenient framework for studying initial value problems of time fractional differential equations. Compared with Riemann-Liouville derivative, the Caputo derivative defined in [8] removes the singularity at $t=0$ and characterizes memory from $t=0_{+}$. It is probably this character that makes the Caputo derivative share many similarities with the corresponding ordinary derivative and then more manageable for Cauchy problems. In this work, we use the Caputo derivative introduced in [8] to investigate the fractional dynamic system (1).

On the other hand, there are many results about time-fractional Navier-Stokes equations, which can be used to simulate anomalous diffusion in fractal media. For instance, applying Laplace and finite Hankel transforms, Chaurasia and Kumar [9] obtained the solution of a time-fractional Navier-Stokes equation. In [10], Zhou and Peng studied the mild solutions of Navier-Stokes equations with a time-fractional derivative, meanwhile Nieto and Planas [11] investigated the existence and uniqueness of mild solutions to the Navier-Stokes equations with time fractional differential operators, and obtained several interesting properties about the solution, such as regularity and decay rate in Lebesgue spaces. Nevertheless, most of the available works including the mentioned ones did not take into account the delay in the external forcing term, and are concerned mainly with the existence of solution/mild solution or the regularity. There is no result on the limit behavior of solutions, even less work about fractional Navier-Stokes equations with delay, such as the existence of weak solution and asymptotical behavior of solutions. Actually, for general fractional PDEs, this discussion is limited due to the lack of tools although some special cases have been studied [12-14].

The traditional method used to study solutions of classic nonlinear PDEs is to find some "a priori" estimates of approximate solutions, then to apply some compactness criteria-i.e., the Arzelà-Ascoli theorem, etc. However, this method seems not to work for fractional PDEs with variable delay. Because of the appearance of variable delay term, the generalized fractional Gronwall inequality [15] (Theorem 1) is not enough to find some "a priori" estimates of Lyapunov functions. Even though Ye and Gao [16] obtained the Henry-Gronwall type retarded integral inequalities, this only works for fractional differential equations with constant delay but not for variable delay. Fortunately, Li and Liu [17] (Theorem 4.1-4.2), generalized the classic Aubin-Lions lemma and some convergence theorem to the fractional case, respectively. To our purpose, we first improve [17] (Proposition 3.5) and [8] (Theorem 4.10). Then, under the condition that $\alpha \in\left(\frac{1}{2}, 1\right)$, we investigate the solutions of our system by combing the Galerkin approximation and the generalized Aubin-Lions lemma as well as the Bellman-Gronwall Lemma.

We would like to mention that Wen, Yu and Wang [18] analyzed the dissipativity of Volterra functional differential equations by using the generalized Halanay inequalities, while Wang and Zou [19] studied the dissipativity and contractivity analysis for fractional functional differential equations and their numerical approximations via a fractional Halanay inequality. However, to analyze the dissipativity of fractional PDEs with variable delay, the fractional Halanay inequality [19] alone is not enough any more, in fact, it cannot be applied directly for our case, either. We modify the fractional Halanay inequality [19] (Lemma 4) to a more general case, and then improve the comparison principle [20] (Lemma 3.4) and combine the fractional Halanay inequality to overcome this difficulty.

Motivated by [19], we study the long time behavior of fractional Navier-Stokes equations with variable delay. More precisely, we first prove the existence and uniqueness of weak solutions by Galerkin approximation, and then analyze the dissipativity of system $(P)$, namely, we obtain the existence of an absorbing set by fractional Halanay inequalities and generalized comparison principle. We would like to mention that similar results about the classic model of problem $(P)$ can be found in [21]. 
The organization of this work is as follows. In the next Section, we recall some basic concepts about fractional calculus, and present some auxiliary lemmas which will be useful in later study. In Section 3, we focus on the existence and uniqueness of weak solutions, and the dissipativity of the fractional dynamic system $(P)$ is shown in Section 4. Throughout the work, $C, c$ are positive constants, which can be different from line to line, even in the same line.

\section{Preliminaries}

In this Section, we first recollect the generalize definitions of fractional calculus to functions valued in general Banach spaces as studied in $[8,17]$. Then we prefer to recall some notations and abstract spaces for the sake of completeness and to make the reading of the paper easier, although the notations and results included in this section may seem somehow repetitive, since they can be found in several already published monographs or articles [22-24]. Besides, two examples of delay are presented and some lemmas, propositions that will be used in our later discussion are stated.

Now, we start with the definition of fractional integral, readers are referred to $[2,3,8]$ for more details.

Definition 1. ([3,17]) The fractional Riemann-Liouville integral of order $\alpha \in(0,1)$ for a function $u: \mathbb{R}^{+} \rightarrow \mathbb{R}$ locally integrable is defined by

$$
\left[I_{\alpha} u\right](t)=\frac{1}{\Gamma(\alpha)} \int_{0}^{t}(t-s)^{\alpha-1} u(s) d s, t>0,
$$

where $\Gamma(\alpha)=\int_{0}^{\infty} x^{\alpha-1} e^{-x} d x$ is the classical Gamma function.

Definition 2. ([8]) Let $X$ be a Banach space. For a locally integrable function $u \in L_{l o c}^{1}((0, T)$; $X)$, if there exists $u_{0} \in X$ such that

$$
\lim _{t \rightarrow 0_{+}} \frac{1}{t} \int_{0}^{t}\left\|u(s)-u_{0}\right\|_{X} d s=0
$$

then $u_{0}$ is called the right limit of $u$ at $t=0$, denote as $u\left(0_{+}\right)=u_{0}$. Similarly, we define $u\left(T^{-}\right)=u_{T}$ to be the left limit of $u$ at $t=T$-i.e., $u_{T} \in X$ such that

$$
\lim _{t \rightarrow T^{-}} \frac{1}{T-t} \int_{t}^{T}\left\|u(s)-u_{T}\right\|_{X} d s=0
$$

As pointed out in [8], this fractional integral can be expressed as the convolution between the kernel $g_{\alpha}(t)=\frac{H(t) t^{\alpha-1}}{\Gamma(\alpha)}$ and $H(t) u(t)$ on $\mathbb{R}$, where

$$
H(t)= \begin{cases}1, & t \geq 0, \\ 0, & t<0 .\end{cases}
$$

is the standard Heaviside step function. By this fact, it is not difficult to verify that the integral operators $I_{\alpha}$ form a semigroup, and $I_{\alpha}$ is a bounded linear operator from $L^{1}(0, T)$ to $L^{1}(0, T)$. Inspired by [25] (Section 5, Chapter 1), Li and Liu [8] proposed a generalized definition of Caputo derivative. The new definition is consistent with various definitions in the literature while revealing the underlying group structure. The underlying group property makes many properties of the Caputo derivative natural. 
Before introducing this generalized Caputo derivative, we need to use the distributions $\left\{g_{\alpha}\right\}$ as the convolution kernels for $\alpha \in(-1,1)$ :

$$
g_{\alpha}(t):=\left\{\begin{array}{l}
\frac{H(t) t^{\alpha-1}}{\Gamma(\alpha)}, \quad \alpha \in(0,1), \\
\delta(t), \quad \alpha=0, \\
\frac{D\left(H(t) t^{\alpha}\right)}{\Gamma(1+\alpha)}, \quad \alpha \in(-1,0),
\end{array}\right.
$$

where $\delta$ is the usual Dirac distribution, and $D$ means the distributional derivative. As in [8], the fractional integral operator $I_{\alpha}$ can be expressed as

$$
\left[I_{\alpha} u\right](t):=g_{\alpha} *[H(t) u(t)]
$$

Given $f, g \in L_{l o c}^{1}(0, T)$, we define the convolution between $f$ and $g$ as

$$
f(t) * g(t)=\int_{0}^{t} f(s) g(t-s) d s .
$$

Now, we introduce the generalized Caputo derivative as

Definition 3. ([8]) Let $\alpha \in(0,1)$. Suppose that $u \in L_{\text {loc }}^{1}(0, T)$ has a right limit $u\left(0_{+}\right)=u_{0}$ at $t=0$ in the sense of Definition 2. The Caputo derivative of fractional order $\alpha$ of $u$ is a distribution in $\mathcal{D}^{\prime}(-\infty, T)$ with support in $[0, T)$, given by

$$
D_{t}^{\alpha} u:=I_{-\alpha} u-u_{0} g_{1-\alpha}=g_{-\alpha} *\left[\left(u-u_{0}\right) H(t)\right]
$$

The right fractional Caputo derivative is defined as

Definition 4. ([17]) Let $\alpha \in(0,1)$. Consider that $u \in L_{\text {loc }}^{1}(-\infty, T)$ has a left limit $u_{T}$ at $t=T$ in the sense of Definition 2. The right Caputo derivative fractional order $\alpha$ of $u$ is a distribution in $\mathcal{D}^{\prime}(\mathbb{R})$ with support in $(-\infty, T]$, given by

$$
\tilde{D}_{c ; T}^{\alpha} u:=\tilde{g}_{-\alpha} *\left[H(T-t)\left(u-u_{T}\right)\right]
$$

To introduce the Caputo derivatives for functions valued in general Banach spaces, for fix $T>0$, we present the following sets:

$$
\mathcal{D}^{\prime}:=\left\{v \mid v: C_{c}^{\infty}((-\infty, T) ; \mathbb{R}) \rightarrow X \text { is a bounded linear operator }\right\},
$$

which is analogous of the distribution $\mathcal{D}^{\prime}$ used in [17]. We would like to point out that $\mathcal{D}^{\prime}$ can be understood as the generalization of distribution. In fact, if $X=\mathbb{R}$, then it is reduced to the usual distribution as in [17].

The weak fractional Caputo derivative of the functions valued in Banach spaces is given by

Definition 5. ([17]) Let $X$ be a Banach space and $u \in L_{l o c}^{1}([0, T) ; X)$. Let $u_{0} \in X$. We define the weak Caputo derivative of fractional order $\alpha$ of $u$ associated with initial value $u_{0}$ to be $D_{t}^{\alpha} u \in \mathcal{D}^{\prime}$ such that for any test function $v \in C_{c}^{\infty}((-\infty, T) ; \mathbb{R})$,

$$
\left\langle v, D_{t}^{\alpha} u\right\rangle:=\int_{-\infty}^{T}\left(\tilde{D}_{c ; T}^{\alpha} v\right)\left(u-u_{0}\right) H(t) d t=\int_{0}^{T}\left(\tilde{D}_{c ; T}^{\alpha} v\right)\left(u-u_{0}\right) d t
$$


Next, let us consider the following usual abstract spaces:

$$
\mathcal{V}=\left\{u \in\left(C_{0}^{\infty}(\Omega)\right)^{2}: \operatorname{div} u=0\right\} .
$$

$H=$ the closure of $\mathcal{V}$ in $\left(L^{2}(\Omega)\right)^{2}$ with norm $|\cdot|$, and inner product $(\cdot, \cdot)$, where for $u, v \in\left(L^{2}(\Omega)\right)^{2}$,

$$
(u, v)=\sum_{j=1}^{2} \int_{\Omega} u_{j}(x) v_{j}(x) d x .
$$

$V=$ the closure of $\mathcal{V}$ in $\left(H_{0}^{1}(\Omega)\right)^{2}$ with norm $\|\cdot\|$, and inner product $((\cdot, \cdot))$, where for $u, v \in\left(H_{0}^{1}(\Omega)\right)^{2}$,

$$
((u, v))=\sum_{i, j=1}^{2} \int_{\Omega} \frac{\partial u_{j}}{\partial x_{i}} \frac{\partial v_{j}}{\partial x_{i}} d x .
$$

It follows that $V \subset H \equiv H^{\prime} \subset V^{\prime}$, where the injections are dense and compact. We will use $\|\cdot\|_{*}$ for the norm in $V^{\prime}$, and $\langle\cdot, \cdot\rangle$ for the duality pairing between $V$ and $V^{\prime}$. Now we define $A: V \rightarrow V^{\prime}$ by $\langle A u, v\rangle=((u, v))$, and the trilinear form $B$ on $V \times V \times V$ by

$$
B(u, v, w)=\sum_{i, j=1}^{2} \int_{\Omega} u_{i} \frac{\partial v_{j}}{\partial x_{i}} w_{j} d x, \forall u, v, w \in V .
$$

Note that the trilinear form $B$ satisfies the following inequalities which will be used later in proofs (see [23] (p. 2015)).

$$
|B(u, v, u)| \leq\|u\|_{\left(L^{4}(\Omega)\right)^{2}}^{2}\|v\| \leq 2^{-1 / 2}|u|\|u\|\|v\|, \quad \forall u, v \in V .
$$

The phase space used in this paper is defined as $C_{H}=C([-h, 0] ; H)$ with the norm

$$
\left\|u_{t}\right\|_{C_{H}}=\sup _{-h \leq \theta \leq 0}|u(t+\theta)|, \text { for } u_{t} \in C_{H} \text { and } t \geq 0,
$$

where $u_{t}$ is a function defined on $[-h, 0]$-i.e., $u_{t}:=u_{t}(\theta)=u(t+\theta), \theta \in[-h, 0]$.

We now enumerate the assumptions on the delay term $g$. For $g:[0, T] \times C_{H} \rightarrow\left(L^{2}(\Omega)\right)^{2}$, we assume:

(g1) For any $\xi \in C_{H}$, the mapping $[0, T] \ni t \mapsto g(t, \xi) \in\left(L^{2}(\Omega)\right)^{2}$ is measurable.

(g2) $\quad g(\cdot, 0)=0$.

(g3) There exists $L_{g}>0$ such that, for any $t \in[0, T]$ and all $\xi, \eta \in C_{H}$,

$$
|g(t, \xi)-g(t, \eta)| \leq L_{g}\|\xi-\eta\|_{C_{H}}
$$

Remark 1. (i) As pointed out in [23], condition (g2) is not a restriction. Indeed, if $|g(\cdot, 0)| \in L^{2}(0, T)$, we could redefine $\hat{l}(t)=l(t)+g(t, 0)$ and $\hat{g}(t, \cdot)=g(t, \cdot)-g(t, 0)$. In this way the problem is exactly the same, $\hat{l}$ and $\hat{g}$ satisfy the required assumptions.

(ii) Conditions (g2) and (g3) imply that

$$
|g(t, \xi)| \leq L_{g}\|\xi\|_{C_{H}}
$$

whence $|g(t, \xi)| \in L^{\infty}(0, T)$. 
Example 1. A forcing term with bounded variable delay. Let $G:[0, T] \times \mathbb{R}^{2} \rightarrow \mathbb{R}^{2}$ be a measurable function satisfying $G(t, 0)=0$ for all $t \in[0, T]$, and assume that there exists $L_{G}>0$ such that

$$
|G(t, u)-G(t, v)|_{\mathbb{R}^{2}} \leq L_{G}|u-v|_{\mathbb{R}^{2}}, \forall u, v \in \mathbb{R}^{2} .
$$

Consider a function $\rho(\cdot):[0,+\infty) \rightarrow[0, h]$, which plays the role of the variable delay. Assume that $\rho(\cdot)$ is measurable and define $g(t, \xi)(x)=G(t, \xi(-\rho(t))(x))$ for each $\xi \in C_{H}, x \in \Omega$ and $t \in[0, T]$. Notice that, in this case, the delayed term $g$ in our problem becomes

$$
g(t, \xi)=G(t, \xi(-\rho(t)))
$$

Example 2. A forcing term with finite distributed delay. Let $G:[0, T] \times[-h, 0] \times \mathbb{R}^{2} \rightarrow \mathbb{R}^{2}$ be a measurable function satisfying $G(t, s, 0)=0$ for all $(t, s) \in[0, T] \times[-h, 0]$, and there exists a function $\beta(s) \in L^{1}(-h, 0)$ such that

$$
|G(t, s, u)-G(t, s, v)|_{\mathbb{R}^{2}} \leq \beta(s)|u-v|_{\mathbb{R}^{2}}, \forall u, v \in \mathbb{R}^{2}, \forall(t, s) \in[0, T] \times[-h, 0]
$$

Define $g(t, \xi)(x)=\int_{-h}^{0} G(t, s, \xi(s)(x))$ ds for each $\xi \in C_{H}, t \in[0, T]$, and $x \in \Omega$. Then, the delayed term $g$ in our problem becomes

$$
g(t, \xi)=\int_{-h}^{0} G(t, s, \xi(s)) d s
$$

After introducing the above operators, an equivalent abstract formulation to problem $(P)$ is

$$
\begin{aligned}
& D_{t}^{\alpha} u+v A u+B(u)=f(t)+g\left(t, u_{t}\right), \quad \forall t>0, \\
& u(t)=\phi(t), t \in[-h, 0] .
\end{aligned}
$$

The definition of weak solution to problem (6) and (7) is defined as

Definition 6. ([17]) Given an initial datum $\phi \in C_{H}$, a weak solution $u$ to (6) and (7) in the interval $[-h, T]$ is a function $u \in C([-h, T] ; H) \cap L^{2}(0, T ; V)$ with $u_{0}=\phi(0)$ such that, for all $v \in V$,

$$
\left(D_{t}^{\alpha} u(t), v\right)+v((u(t), v))+B(u(t), u(t), v)=\langle f(t), v\rangle+\left(g\left(t, u_{t}\right), v\right),
$$

where the equation must be understood in the sense of distribution.

The following auxiliary Lemmas will be needed in this work.

Lemma 1. (See $[17,26])$ For any function $u(t)$ absolutely continuous on $[0, T]$, one has the inequality

$$
u(t) D_{t}^{\alpha} u(t) \geq \frac{1}{2} D_{t}^{\alpha} u^{2}(t), \alpha \in(0,1)
$$

The following result is a generalization of the Aubin-Lions Lemma [27].

Lemma 2. ([17] (Theorem 4.2)) Let $T>0, \alpha \in(0,1)$ and $p \in[1, \infty)$. Let $M, X, Y$ be Banach spaces. The inclusion $M \hookrightarrow X$ compact and the inclusion $X \hookrightarrow Y$ continuous. Suppose $W \subset L_{\text {loc }}^{1}((0, T) ; M)$ satisfies: 
(i) There exists $r_{1} \in[1, \infty)$ and $C>0$ such that $\forall u \in W$,

$$
\sup _{t \in(0, T)} I^{\alpha}\left(\|u\|_{M}^{r_{1}}\right)=\sup _{t \in(0, T)} \frac{1}{\Gamma(\alpha)} \int_{0}^{t}(t-s)^{\alpha-1}\|u\|_{M}^{r_{1}}(s) d s \leq C .
$$

(ii) There exists $p_{1} \in(p, \infty]$, such that, $W$ is bounded in $L^{p_{1}}((0, T) ; X)$.

(iii) There exists $r_{2} \in[1, \infty), C>0$ such that for any $u \in W$ with right limit $u_{0}$ at $t=0$, it holds that

$$
\left\|D_{t}^{\alpha} u\right\|_{L^{r_{2}((0, T) ; Y)}} \leq C .
$$

Then, $W$ is relatively compact in $L^{p}((0, T) ; X)$.

Proposition 1. (An improvement in [17] (Proposition 3.5)) Suppose $Y$ is a reflexive Banach space, $\alpha \in(0,1)$ and $T>0$. Assume the sequence $\left\{u^{n}\right\}$ converges to $u$ in $L^{p}((0, T) ; Y), p \geq 1$. If there is an assignment of initial values $u_{0, n}$ for $u^{n}$ such that the weak Caputo derivatives $D_{t}^{\alpha} u^{n}$ are bounded in $L^{r}((0, T) ; Y)(r \in[1, \infty))$, then

(i) There is a subsequence such that $u_{0, n}$ converges weakly to some value $u_{0} \in Y$.

(ii) If $r>1$, there exists a subsequence such that $D_{t}^{\alpha} u^{n k}$ converges weakly to $v$ and $u_{0, n_{k}}$ converges weakly to $u_{0}$. Moreover, $v$ is the Caputo derivative of $u$ with initial value $u_{0}$ so that

$$
u(t)=u_{0}+\frac{1}{\Gamma(\alpha)} \int_{0}^{t}(t-s)^{\alpha-1} v(s) d s .
$$

Further, if $r \geq 1$, then, $u\left(0_{+}\right)=u_{0}$ in $Y$ is the sense of Definition 2 .

Proof. We would like to mention that this Proposition is just a slightly improvement of [17] (Proposition 3.5), in which, the final conclusion-i.e., $u\left(0_{+}\right)=u_{0}$ in $Y$-holds true for $r \geq \frac{1}{\alpha}$. However, this conclusion holds for $r \geq 1$.

So, we just need to prove that for $r \geq 1$, if $D_{t}^{\alpha} u \in L_{l o c}^{1}([0, T), Y)$, then $u\left(0_{+}\right)=u_{0}$ in $Y$ under the sense of Definition 2. By a similar argument in [17] (Corollary 2.16) and Young's inequality with the conjugate index $p=\infty, q=1, \frac{1}{p}+\frac{1}{q}=1$, we find

$$
\begin{aligned}
\frac{1}{t} \int_{0}^{t}\left\|u-u_{0}\right\|_{Y} d t & \leq \frac{1}{t \Gamma(\alpha)} \int_{0}^{t} \int_{0}^{\tau}(\tau-s)^{\alpha-1}\left\|D_{t}^{\alpha} u\right\|_{Y} d s d \tau \\
& \leq \frac{1}{t \Gamma(\alpha+1)} \int_{0}^{t}(t-s)^{\alpha}\left\|D_{t}^{\alpha} u\right\|_{Y} d s \\
& \leq \frac{1}{\Gamma(\alpha+2)} t^{\alpha}\left\|D_{t}^{\alpha} u\right\|_{L^{1}((0, t), Y)} \rightarrow 0 \text { as } t \rightarrow 0_{+}
\end{aligned}
$$

Since $\left\|D_{t}^{\alpha} u\right\|_{Y}$ is integrable on $[0, T-\delta]$ for some $\delta>0$. The proof is finished immediately.

Remark 2. Li and Liu in [17] (Theorem 5.2) proved the existence of weak solution for a time fractional incompresible Navier-Stokes equation for $\alpha \in\left[\frac{1}{2}, 1\right)$, because $u\left(0_{+}\right)=u_{0}$ is obtained under this condition. However, by using this Proposition 1, we also can prove $u\left(0_{+}\right)=u_{0}$ for $\alpha \in(0,1)$. Therefore, the existence result of [17] (Theorem 5.2) still holds for $\alpha \in(0,1)$. In this extent, we say that Proposition 1 improves [17] (Proposition 3.5). 
Proposition 2. (Modified Fractional Halanay Inequality) Assume that the non-negative continuous function $v$ satisfies

$$
\begin{aligned}
& D_{t}^{\alpha} v(t) \leq \gamma+a v(t)+b \sup _{t-\tau(t) \leq s \leq t} v(s), 0<t \leq T, \\
& v(t)=|\varphi(t)|, \quad-\sigma \leq t \leq 0
\end{aligned}
$$

where $\gamma$ is a positive constant and $a+b \neq 0, \sigma=-\inf _{t \geq 0}(t-\tau(t))>0$, and the delay function $\tau(t) \geq 0$. If $a+b<0$, then the following estimates holds

$$
v(t) \leq-\frac{\gamma}{a+b}+M^{\prime} E_{\alpha}\left(\lambda^{*} t^{\alpha}\right), \text { for all } t \geq \tau(t),
$$

where $M^{\prime}=\sup _{-h \leq t \leq 0}|\varphi(t)|$, and the parameter $\lambda^{*}$ is defined by

$$
\lambda^{*}=\sup _{t-\tau(t) \geq 0}\left\{\lambda: \lambda-a-b \frac{E_{\alpha}\left(\lambda(t-\tau(t))^{\alpha}\right)}{E_{\alpha}\left(\lambda t^{\alpha}\right)}=0\right\}
$$

and it holds that $\lambda^{*} \in[a+b, 0]$.

Further, if the delay is bounded-i.e., $\tau(t) \leq \tau_{0}$ for some constant $\tau_{0}$ - then the parameter $\lambda^{*}$ defined by

$$
\lambda^{*}=\sup _{t-\tau(t) \geq 1}\left\{\lambda: \lambda-a-b \frac{E_{\alpha}\left(\lambda(t-\tau(t))^{\alpha}\right)}{E_{\alpha}\left(\lambda t^{\alpha}\right)}=0\right\}
$$

is strictly negative, namely, there exists some positive constants $\epsilon_{0}$ satisfying $a+b<-\epsilon_{0}$ such that $\lambda^{*} \in\left[a+b,-\epsilon_{0}\right]$, and the estimate in (9) holds for all $t$ such that $t \geq \tau(t)+1$.

Proof. Actually, Proposition 2 is a slightly modification of [19] (Lemma 4), in which $\tau(t)>0$ strictly for the first conclusion (9). However, in our case, (9) holds true for $\tau(t) \geq 0$. So, we only need to prove (9) is true when $\tau(t)=0$. We prove this by comparison principle.

If $\tau(t)=0$, then the original system (8) becomes.

$$
\begin{aligned}
& D_{t}^{\alpha} v(t) \leq \gamma+(a+b) v(t), \quad 0<t \leq T, \\
& v(0)=|\varphi(0)|, \quad t=0
\end{aligned}
$$

where $\gamma$ is a positive constant and $a+b<0$.

From system (8), there exists a nonnegative function $m(t)$ satisfying

$$
\begin{aligned}
& D_{t}^{\alpha} v(t)=\gamma+(a+b) v(t)+m(t), \quad 0<t \leq T, \\
& v(0)=|\varphi(0)|, \quad t=0 .
\end{aligned}
$$


According to [2] (Theorem 4.3), the initial value problem (8) has a unique solution that can be represented by

$$
\begin{aligned}
v(t) & =|\phi(0)| E_{\alpha}\left((a+b) t^{\alpha}\right)+\int_{0}^{t}(t-s)^{\alpha-1} E_{\alpha, \alpha}\left((a+b)(t-s)^{\alpha}\right)(\gamma+m(s)) d s \\
& \leq|\phi(0)| E_{\alpha}\left((a+b) t^{\alpha}\right)+\gamma \int_{0}^{t}(t-s)^{\alpha-1} E_{\alpha, \alpha}\left((a+b)(t-s)^{\alpha}\right) d s \\
& \leq|\phi(0)| E_{\alpha}\left((a+b) t^{\alpha}\right)-\frac{\gamma}{a+b} \\
& \leq M^{\prime} E_{\alpha}\left(\lambda^{*} t^{\alpha}\right)-\frac{\gamma}{a+b}
\end{aligned}
$$

where we used that $t^{\alpha-1}$ and $E_{\alpha, \alpha}\left((a+b) t^{\alpha}\right)$ are nonnegative and $\lambda^{*} \in[a+b, 0]$, as well as the fact that $E_{\alpha}\left(\lambda t^{\alpha}\right)$ is non-decreasing respect to $\lambda$. The proof is complete.

Remark 3. It turns out that the modified fractional Halanay inequality holds true not only for delay fractional dynamical system but also for the nondelay case, which means that it could be applied to more fractional differential equations. In this sense, we say it improves [19] (Lemma 4).

Proposition 3. (The generalized comparison principle.) Assume that for any function $u$ and $w$ are absolutely continuous on $[0, T]$, one has the inequality

$$
\begin{aligned}
& D_{t}^{\alpha} u(t) \leq-a u(t)+b u(t-\tau(t))+c, 0<t<T, \\
& u(t)=\varphi(t), \quad-h \leq t \leq 0,
\end{aligned}
$$

and the following fractional differential equation

$$
\begin{aligned}
& D_{t}^{\alpha} w(t)=-a w(t)+b w(t-\tau(t))+c, 0<t<T, \\
& w(t)=\varphi(t), \quad-h \leq t \leq 0,
\end{aligned}
$$

where $a, b, c$ are positive constants. Then it holds that

$$
u(t) \leq w(t), \text { for all } t \geq-h
$$

Proof. Obviously, (12) holds true for any $t \in[-h, 0]$. Hence, we only need to verify that (12) is correct for $t \in[0, T]$. We will prove this through two steps.

Step 1. We first prove that (12) holds for $t>\tau(t)$. By contradiction, if it is not true, then there exists some $t>\tau(t)$ such that $u(t) \geq w(t)$. Denote $t_{*}$ by

$$
t_{*}=\inf \{t>\tau(t): u(t) \geq w(t)\}
$$

Now, set $z(t)=u(t)-w(t)$. Then we know from the definition that $z\left(t_{*}\right)=0$, and $z(t)<0$ for $0<t_{*}-\tau\left(t_{*}\right) \leq t<t_{*}$. Then by the fractional comparison principle in [19] (Lemma 3), we have that

$$
D_{t}^{\alpha} z\left(t_{*}\right) \geq 0
$$


However,

$$
\begin{aligned}
D_{t}^{\alpha} z\left(t_{*}\right) & =D_{t}^{\alpha} u\left(t_{*}\right)-D_{t}^{\alpha} w\left(t_{*}\right) \\
& \leq-a\left(u\left(t_{*}\right)-w\left(t_{*}\right)\right)+b\left(u\left(t_{*}-\tau\left(t_{*}\right)\right)-w\left(t_{*}-\tau\left(t_{*}\right)\right)\right) \\
& =-a z\left(t_{*}\right)+b z\left(t_{*}-\tau\left(t_{*}\right)\right) \leq b z\left(t_{*}-\tau\left(t_{*}\right)\right)<0
\end{aligned}
$$

which contradicts (13); therefore, $u(t) \leq w(t)$ for $t>\tau(t)$.

Step 2. On the other hand, when $0<t \leq \tau(t)$, then $-h \leq t-\tau(t) \leq 0$, since $\tau(t) \in[0, h]$. So (14) and (15) can be rewritten as, respectively,

$$
\begin{aligned}
& D_{t}^{\alpha} u(t) \leq-a u(t)+b \varphi(t-\tau(t))+c, 0<t \leq \tau(t), \\
& u(t)=\varphi(t),-h \leq t \leq 0,
\end{aligned}
$$

and the following fractional differential equation

$$
\begin{aligned}
& D_{t}^{\alpha} w(t)=-a w(t)+b \varphi(t-\tau(t))+c, 0<t \leq \tau(t), \\
& w(t)=\varphi(t), \quad-h \leq t \leq 0,
\end{aligned}
$$

Then there is a nonnegative function $m(t)$, such that

$$
\begin{aligned}
& D_{t}^{\alpha} u(t)=-a u(t)+b \varphi(t-\tau(t))-m(t)+c, 0<t \leq \tau(t), \\
& u(t)=\varphi(t), \quad-h \leq t \leq 0
\end{aligned}
$$

Then, by [28] (Theorem 1), system (16) has a unique solution on $[0, h]$ that can be represented as

$$
u(t)=\int_{0}^{t}(t-s)^{\alpha-1} E_{\alpha, \alpha}\left(-a(t-s)^{\alpha}\right)(b \varphi(s-\tau(s))-m(s)+c) d s+c^{\prime} E_{\alpha}\left(-a t^{\alpha}\right), 0<t \leq \tau(t) .
$$

Similarly, the solution of system (15) can be written as

$$
w(t)=\int_{0}^{t}(t-s)^{\alpha-1} E_{\alpha, \alpha}\left(-a(t-s)^{\alpha}\right)(b \varphi(s-\tau(s))+c) d s+c^{\prime} E_{\alpha}\left(-a t^{\alpha}\right), 0<t \leq \tau(t) .
$$

Notice that $t^{\alpha}$ and $E_{\alpha, \alpha}\left(-a t^{\alpha}\right)$ are non-negative for $a>0$, then we have $u(t) \leq w(t)$ for all $0<t \leq \tau(t)$. In summary, $u(t) \leq w(t)$ for all $t \geq-h$.

Therefore the proof is complete.

Remark 4. We would like to point out that Proposition 3 generalizes the conclusion in [8] (Theorem 4.10) to some extent. Proposition 3 also improves the comparison principle in [20] (Lemma 3.4), which is proven only for constant delay-i.e., $\tau(t)=\tau$. However, in our case, the delay term $\tau(t)$ is a function taking values in $[0, h]$. In this way, we could say that Lemma 3.4 of [20] is a special case of Proposition 3.

Lemma 3. (Bellman-Gronwall Lemma [29] (p. 252)) Let $T>0, g \in L^{1}(0, T)$ and $g \geq 0$ a.e., $C_{1}, C_{2}$ be positive constants. If $\varphi \in L^{1}(0, T), \varphi \geq 0$ a.e., satisfying $g \varphi \in L^{1}(0, T)$ and

$$
\varphi(t) \leq C_{1}+C_{2} \int_{0}^{t} g(s) \varphi(s) d s, \text { a.e. } t \in(0, T),
$$


then

$$
\varphi(t) \leq C_{1} \exp \left\{C_{2} \int_{0}^{t} g(s) d s\right\}, \text { a.e. } t \in(0, T)
$$

Remark 5. Actually, the positive constants $C_{1}, C_{2}$ can be replaced by functions $C_{1}(t)$ or $C_{2}(t)$, but a similar result can be obtained-readers are referred to [29] (p. 252) for more information.

\section{Existence and Uniqueness of Weak Solutions}

In this section, we prove the existence and uniqueness of weak solutions to problem (6) and (7) by Galerkin approximations. Denote

$$
\lambda_{1}=\inf _{v \in V \backslash\{0\}} \frac{\|v\|^{2}}{|v|^{2}}>0 .
$$

Moreover,

$$
\int_{0}^{t}\|f(s)\|_{*}^{2\left(1+\frac{1}{\alpha}\right)} d s<\infty, \quad \alpha \in(0,1), \text { for any } t \geq 0 .
$$

We have the following result:

Theorem 1. Suppose that $(g 1)-(g 3)$ and (17) hold true, then for any $\phi \in C_{H}$ and $\alpha \in\left(\frac{1}{2}, 1\right)$ system, (6) and (7) has a unique weak solution.

Proof. We split it into several steps.

Step 1. The Galerkin approximation. By the definition of $A=-\Delta$ and the classical spectral theory of elliptic operators, it follows that $A$ possesses a sequence of eigenvalues $\left\{\lambda_{j}\right\}_{j \geq 1}$ and a corresponding family of eigenfunctions $\left\{w_{j}\right\}_{j \geq 1} \subset V$, which form a Hilbert basis of $H$, dense on $V$. We consider the subspace $V_{m}=\operatorname{span}\left\{w_{1}, w_{2}, \cdots, w_{m}\right\}$, and the projector $P_{m}: H \rightarrow V_{m}$ given by $P_{m} u=\sum_{j=1}^{m}\left(u, w_{j}\right) w_{j}$, and define $u^{(m)}(t)=\sum_{j=1}^{m} \gamma_{m, j}(t) w_{j}$, where the superscript $m$ will be used instead of $(m)$, for short, since no confusion is possible with powers of $u$, and where the coefficients $\gamma_{m, j}(t)$ are required to satisfy the Cauchy problem

$$
\begin{aligned}
& \left(D_{t}^{\alpha} u^{m}(t), w_{j}\right)+v\left(\left(u^{m}(t), w_{j}\right)\right)+B\left(u^{m}(t), u^{m}(t), w_{j}\right)=\left\langle f(t), w_{j}\right\rangle+\left(g\left(t, u_{t}^{m}\right), w_{j}\right), 1 \leq j \leq m, \\
& u^{m}(t)=P_{m} \phi(t), t \in[-h, 0] .
\end{aligned}
$$

The above system of fractional order functional differential equations with finite delay fulfills the conditions for the existence and uniqueness of a local solution (e.g., cf. [30] (Theorem 3.1)). Hence, we conclude that (18) has a unique local solution defined in $\left[0, t_{m}\right)$ with $0 \leq t_{m} \leq T$. Next, we will obtain a priori estimates and ensure that the solutions $u^{m}$ do exist in the whole interval $[0, \infty)$. Assume that $M=\|\phi\|_{C_{H}}^{2}=\sup _{-h \leq t \leq 0}|\phi(t)|^{2}$.

Step 2. A priori estimates. Multiplying (18) by $\gamma_{m, j}(t), j=1, \ldots, m$, summing up, and using Lemma 1 , Cauchy-Schwartz and Young's inequalities, we obtain

$$
\begin{aligned}
\frac{1}{2} D_{t}^{\alpha}\left|u^{m}(t)\right|^{2}+v\left\|u^{m}(t)\right\|^{2} & \leq\|f(t)\| *\left\|u^{m}(t)\right\|+\left|g\left(u_{t}^{m}\right) \| u^{m}(t)\right| \\
& \leq \frac{v}{2}\left\|u^{m}(t)\right\|^{2}+\frac{\|f(t)\|_{*}^{2}}{2 v}+L_{g}\left\|u_{t}^{m}\right\|_{C_{H}}^{2} .
\end{aligned}
$$

Hence, 


$$
D_{t}^{\alpha}\left|u^{m}(t)\right|^{2}+v\left\|\nabla u^{m}(t)\right\|^{2} \leq \frac{\|f(t)\|_{*}^{2}}{v}+2 L_{g}\left\|u_{t}^{m}\right\|_{C_{H}}^{2}
$$

Multiplying (19) by $I_{\alpha}$, and let $p=1+\alpha, q=1+\frac{1}{\alpha}$, we find

$$
\begin{aligned}
& \left|u^{m}(t)\right|^{2}+\frac{v}{\Gamma(\alpha)} \int_{0}^{t}(t-s)^{\alpha-1}\left\|\nabla u^{m}(s)\right\|^{2} d s \\
& \leq\left|u^{m}(0)\right|^{2}+\frac{1}{v \Gamma(\alpha)} \int_{0}^{t}(t-s)^{\alpha-1}\|f(s)\|_{*}^{2} d s+\frac{2 L_{g}}{\Gamma(\alpha)} \int_{0}^{t}(t-s)^{\alpha-1}\left\|u_{s}^{m}\right\|_{C_{H}}^{2} d s \\
& \leq\left|u^{m}(0)\right|^{2}+\frac{1}{v \Gamma(\alpha)}\left(\int_{0}^{t}(t-s)^{p(\alpha-1)} d s\right)^{\frac{1}{p}}\left(\int_{0}^{t}\|f(s)\|_{*}^{2 q} d s\right)^{1 / q}+\frac{2 L_{g}}{\Gamma(\alpha)} \int_{0}^{t}(t-s)^{\alpha-1}\left\|u_{s}^{m}\right\|_{C_{H}}^{2} d s \\
& \leq\left|u^{m}(0)\right|^{2}+\frac{1}{v \alpha^{2} \Gamma(\alpha)} t^{\alpha^{2}} F(t)+\frac{2 L_{g}}{\Gamma(\alpha)}\left(\int_{0}^{t}(t-s)^{p(\alpha-1)} e^{p s} d s\right)^{\frac{1}{p}}\left(\int_{0}^{t} e^{-q s}\left\|u_{s}^{m}\right\|_{C_{H}}^{2 q} d s\right)^{1 / q} \\
& \leq\left|u^{m}(0)\right|^{2}+\frac{1}{v \alpha^{2} \Gamma(\alpha)} t^{\alpha^{2}} F(t)+\frac{2 L_{g} \Gamma\left(\alpha^{2}\right)}{\Gamma(\alpha)}(1+\alpha)^{-\frac{\alpha^{2}}{1+\alpha}} e^{t}\left(\int_{0}^{t} e^{-q s}\left\|u_{s}^{m}\right\|_{C_{H}}^{2 q} d s\right)^{1 / q} .
\end{aligned}
$$

Denote by $A(t)=\left|u^{m}(0)\right|^{2}+\frac{1}{v \alpha^{2} \Gamma(\alpha)} t^{\alpha^{2}} F(t), B(t)=\frac{2 L_{g} \Gamma\left(\alpha^{2}\right)}{\Gamma(\alpha)}(1+\alpha)^{-\frac{\alpha^{2}}{1+\alpha}} e^{t}$. Then, we have

$$
\left\|u_{t}^{m}\right\|_{\mathcal{C}_{H}}^{2}+v \int_{0}^{t+\theta}(t+\theta-s)^{\alpha-1}\left\|\nabla u^{m}(s)\right\|^{2} d s \leq A(t)+B(t)\left(\int_{0}^{t}\left\|u_{s}^{m}\right\|^{2 q} d s\right)^{1 / q} .
$$

Therefore,

$$
\left\|u_{t}^{m}\right\|_{C_{H}}^{2 q} \leq 2^{q} A^{q}(t)+2^{q} B^{q}(t)\left(\int_{0}^{t}\left\|u_{s}^{m}\right\|_{C_{H}}^{2 q} d s\right)
$$

Using the Gronwall Lemma, we obtain that

$$
\left\|u_{t}^{m}\right\|_{\mathcal{C}_{H}}^{2} \leq c\left(A(t)+B(t) \int_{0}^{t} A(s) e^{c \int_{s}^{t} B(r) d r} d s\right), \text { for all } t \in[0, T] \text { and } \theta \in[-h, 0] .
$$

Hence, we conclude that for any $T>0,\left\|u_{t}^{m}\right\|_{C_{H}}$ is finite, which means the local solution $u^{m}(t ; \phi)$ is actually a global one. We also can have that there exists a constant $C>0$, depending on some constants of the problem (namely, $v, L_{g}$ and $f$ ), and on $T$ and $M>0$, such that

$$
\left\|u_{t}^{m}\right\|_{C_{H}}^{2} \leq C(T, M) \forall t \in[0, T],\|\phi\|_{C_{H}} \leq M, \forall m \geq 1,
$$

which also implies that $\left\{u^{m}\right\}$ is bounded in $L^{\infty}(-h, T ; H)$.

Now it follows from (20) and the above uniform estimates that

$$
\begin{aligned}
v\left\|u^{m}\right\|_{L^{2}(0, T ; V)}^{2} & \leq v t^{1-\alpha} \int_{0}^{t}(t-s)^{\alpha-1}\left\|u^{m}(s)\right\|^{2} d s \\
& \leq t^{1-\alpha}\left(\Gamma(\alpha)\left|u^{m}(0)\right|^{2}+\int_{0}^{t}(t-s)^{\alpha-1}\left(\frac{1}{v}\|f(s)\|_{*}^{2}+2 L_{g} C(T, M)\right) d s\right) \\
& \leq C(T, M), \quad \forall m \geq 1 .
\end{aligned}
$$


Therefore, we conclude that

$$
\left\{u^{m}\right\} \text { is bounded in } L^{2}((0, T) ; V) \cap L^{\infty}((-h, T) ; H) .
$$

From (5) and (18), it holds $\left\|D_{t}^{\alpha} u^{m}\right\|_{*} \leq v\left\|u^{m}\right\|+2^{1 / 2}\left|u^{m}\right| \cdot\left\|u^{m}\right\|+\|f\|_{*}+\lambda_{1}^{-1 / 2}\left|g\left(t, u_{t}^{m}\right)\right|$, which implies that

$$
\left\{D_{t}^{\alpha} u^{m}\right\} \text { is bounded in } L^{2}\left(0, T ; V^{\prime}\right) \text {. }
$$

Step 3. Approximation of initial datum in $C_{H}$. Let us check

$$
P_{m} \phi \rightarrow \phi \text { in } C_{H}
$$

Assume that $\theta_{m} \rightarrow \theta \in[-h, 0]$, then $P_{m} \phi\left(\theta_{m}\right) \rightarrow \phi(\theta)$, since $\left\|P_{m} \phi\left(\theta_{m}\right)-\phi(\theta)\right\| \leq \| P_{m} \phi\left(\theta_{m}\right)-$ $P_{m} \phi(\theta)\|+\| P_{m} \phi(\theta)-\phi(\theta) \| \rightarrow 0$ as $m \rightarrow \infty$. So (23) holds true.

Step 4. Compactness results. By (21) and (22), the compact imbedding $V \hookrightarrow H$, and the generalized Aubin-Lions Lemma 2 as well as Proposition 1, for any $\alpha \in(0,1)$, we obtain there exist a subsequence still relabeled as $\left\{u^{m}\right\}$ and function $u \in C([-h, T) ; H) \cap L^{2}((0, T) ; V)$ for all $T>0$, with $u(t)=\phi(t)$ in $[-h, 0], u\left(0_{+}\right)=u_{0}$, and $D_{t}^{\alpha} u \in L^{2}\left((0, T) ; V^{\prime}\right)$ for all $T>0$, and an element $\chi \in L^{\infty}((0, T) ; H)$ such that

$$
\begin{aligned}
& u^{m} \stackrel{*}{\rightarrow} u \text { weakly-star in } L^{\infty}((0, T) ; H), \\
& u^{m} \rightarrow u \text { weakly in } L^{2}((0, T) ; V), \\
& D_{t}^{\alpha} u^{m} \rightarrow D_{t}^{\alpha} u \text { weakly in } L^{2}\left((0, T) ; V^{\prime}\right), \\
& u^{m} \rightarrow u \text { strongly in } L^{2}((0, T) ; H), \\
& g\left(\cdot, u_{t}^{m}\right) \stackrel{*}{\rightarrow} \chi \text { weakly-star in } L^{\infty}((0, T) ; H) .
\end{aligned}
$$

Observe that if $\alpha \in\left(\frac{1}{2}, 1\right)$, for all $s, t \in[0, T]$, by

$$
\begin{aligned}
u^{m}(t)-u^{m}(s) & =\frac{1}{\Gamma(\alpha)} \int_{s}^{t}(t-r)^{\alpha-1}\left(D_{r}^{\alpha} u^{m}\right)(r) d r \\
& \leq \frac{1}{\Gamma(\alpha)(2 \alpha-1)}(t-s)^{2 \alpha-1}\left\|D_{t}^{\alpha} u^{m}\right\|_{L^{2}\left((s, t), V^{\prime}\right)}, \text { in } V^{\prime},
\end{aligned}
$$

and combing (22) we find that $u^{m}$ is equi-continuous on $[0, T]$ with values in $V^{\prime}$. Notice that the inclusion $H \hookrightarrow V^{\prime}$ compact, so using Ascoli-Arzelà and (24), we conclude that

$$
u^{m} \rightarrow u \text { in } C\left([0, T] ; V^{\prime}\right), \forall T>0 .
$$

Combining (21) and (25), then for any $\left\{t_{m}\right\} \subset[0, T)$ with $t_{m} \rightarrow t$, one obtains

$$
u^{m}\left(t_{m}\right) \rightarrow u(t) \text { weakly in } H .
$$

Now we prove that

$$
u^{m} \rightarrow u \text { in } C([0, T] ; H), \forall T>0 .
$$

By contradiction, if (27) is not true, then there would exists a $\epsilon_{1}>0,\left\{t_{m}\right\}$ and $t_{*}$ with $t_{m} \rightarrow t_{*}$ such that

$$
\left|u^{m}\left(t_{m}\right)-u\left(t_{*}\right)\right| \geq \epsilon_{1} .
$$


On one hand, by (26), we have $\left|u\left(t_{*}\right)\right| \leq \lim _{n \rightarrow \infty} \inf \left|u^{m}\left(t_{m}\right)\right|$. Therefore, if we could prove $\left|u^{m}\left(t_{m}\right)\right| \rightarrow$ $\left|u\left(t_{*}\right)\right|$, then (28) is contradictory, in other words, (27) is obtained immediately. To this end, it is enough to show that

$$
\lim _{m \rightarrow \infty} \sup \left|u^{m}\left(t_{m}\right)\right| \leq\left|u\left(t_{*}\right)\right| .
$$

On the other hand, for system (18), we have the following energy inequality,

$$
\begin{aligned}
& \left|u^{m}(t)\right|^{2}+\frac{v}{\Gamma(\alpha)} \int_{0}^{t}(t-s)^{\alpha-1}\left\|u^{m}(s)\right\|^{2} d s \\
& \leq\left|u^{m}(0)\right|^{2}+\frac{1}{\Gamma(\alpha)} \int_{0}^{t}(t-s)^{\alpha-1}\|f(s)\|_{*}^{2} d s+\frac{2 L_{g} C(T, M)}{\Gamma(\alpha+1)}(t-s)^{\alpha} .
\end{aligned}
$$

Besides, by (24), passing to the limit in (18), we have that $u \in C([0, T] ; H)$ is a solution of a similar problem to (6)-i.e.,

$$
\left(D_{t}^{\alpha} u, v\right)+v(A u, v)+(B(u), v)=\langle f(t), v\rangle+(\chi, v), \quad \forall v \in V^{\prime}
$$

which also has the energy inequality,

$$
\begin{aligned}
& |u(t)|^{2}+\frac{v}{\Gamma(\alpha)} \int_{0}^{t}(t-s)^{\alpha-1}\|u(s)\|^{2} d s \\
& \leq|u(0)|^{2}+\frac{1}{\Gamma(\alpha)} \int_{0}^{t}(t-s)^{\alpha-1}\|f(s)\|_{*}^{2} d s+\frac{1}{\Gamma(\alpha)} \int_{0}^{t}(t-s)^{\alpha-1}(\chi(s), u(s)) d s .
\end{aligned}
$$

Combing the last convergence in (24) and the dominate convergence theorem, we find

$$
\int_{0}^{t}(t-s)^{\alpha-1}|\chi(s)|^{2} d s \leq \lim _{m \rightarrow \infty} \inf \int_{0}^{t}(t-s)^{\alpha-1}\left|g\left(u_{s}^{m}\right)\right|^{2} d s \leq \frac{2 L_{g}}{\alpha} C(T, M)(t-s)^{\alpha} .
$$

Therefore, $u$ also satisfies inequality (30) with the same last term on the right-hand side. Consider now two continuous functions defined as

$$
\begin{aligned}
& J(t)=|u(t)|^{2}-\frac{1}{\Gamma(\alpha)} \int_{0}^{t}(t-s)^{\alpha-1}\|f(s)\|_{*}^{2} d s-\frac{2 L_{g} C(T, M)}{\Gamma(\alpha+1)}(t-s)^{\alpha}, \\
& J_{m}(t)=\left|u^{m}(t)\right|^{2}-\frac{1}{\Gamma(\alpha)} \int_{0}^{t}(t-s)^{\alpha-1}\|f(s)\|_{*}^{2} d s-\frac{2 L_{g} C(T, M)}{\Gamma(\alpha+1)}(t-s)^{\alpha} .
\end{aligned}
$$

$J$ and $J_{m}$ are non-increasing in $t$. Moreover, again from (24), we have

$$
J_{m}(t) \rightarrow J(t) \text {, a.e. } t \in(0, T) .
$$

Assume that $t_{*}>0$, consider $\left\{t_{k}\right\} \subset\left(0, t_{*}\right)$ with $t_{k} \rightarrow t_{*}$, by the continuity of $J$,

$$
\exists k_{\epsilon}:\left|J\left(t_{k}\right)-J\left(t_{*}\right)\right|<\frac{\epsilon}{2}, \forall k \geq k_{\epsilon} .
$$

Take now $m\left(k_{\epsilon}\right)$ such that

$$
t_{m} \geq t_{k_{\epsilon}}:\left|J_{m}\left(t_{k_{\epsilon}}\right)-J\left(t_{k_{\epsilon}}\right)\right|<\frac{\epsilon}{2}, \forall n \geq n\left(k_{\epsilon}\right) .
$$


Then, we conclude that for all $m \geq n\left(k_{\epsilon}\right)$

$$
J_{m}\left(t_{m}\right)-J\left(t_{*}\right) \leq\left|J_{m}\left(t_{k_{\epsilon}}\right)-J\left(t_{k_{\epsilon}}\right)\right|+\left|J\left(t_{k_{\epsilon}}\right)-J\left(t_{*}\right)\right|<\epsilon,
$$

which gives (29).

Therefore, we find that

$$
u^{m} \rightarrow u \text { in } C([0, T] ; H)
$$

Then, steps 3 and 4 imply that

$$
u_{t}^{m} \rightarrow u_{t} \text { in } C_{H}, \forall 0 \leq t \leq T
$$

Therefore, combining $(g 3)$, we can prove that

$$
g\left(\cdot, u_{.}^{m}\right) \rightarrow g(\cdot, u .) \text { in } L^{2}(0, T ; H) .
$$

Thus, we can finally pass to the limit in (18), concluding that $u$ solves $(P)$.

Step 5. Uniqueness of solution. Let $u(t ; \phi), v(t ; \phi)$ be two solutions of $(P)$ with the same initial values-i.e., $u(t)=v(t)=\phi(t), t \in[-h, 0]$. Set $w(t)=u(t)-v(t), t \geq 0$, then $w(t)=0$, for all $t \in[-h, 0]$. For $w(t)$, we have

$$
D_{t}^{\alpha} w-v \Delta w+B(u)-B(v)=g\left(t, u_{t}\right)-g\left(t, v_{t}\right)
$$

Multiplying above equation by $w(t)$, and integral over $\Omega$, we obtain

$$
\begin{aligned}
D_{t}^{\alpha}|w|^{2}+v\|w\|^{2} & =-(B(u)-B(v), w)+\left(g\left(t, u_{t}\right)-g\left(t, v_{t}\right), w\right) \\
& \leq c|w|^{2}\|v\|^{2}+2 L_{g} \sup _{0 \leq s \leq t}|w(s)|^{2} \\
& \leq c\left(\|v\|^{2}+1\right) \sup _{0 \leq s \leq t}|w(s)|^{2}, \text { for all } t \in[0, T] .
\end{aligned}
$$

The above inequality holds true for any $t \in[0, T]$, then we have

$$
\sup _{0 \leq s \leq t}|w(s)|^{2} \leq|w(0)|^{2}+c \int_{0}^{t}\left(\|v\|^{2}+1\right)(t-s)^{\alpha-1} \sup _{0 \leq r \leq s}|w(r)|^{2} d s .
$$

Using the Bellman-Gronwall Lemma 3 and (21), we have

$$
\sup _{0 \leq s \leq t}|w(s)|^{2} \leq|w(0)|^{2} \exp \left\{c \int_{0}^{t}\left(\|v\|^{2}+1\right)(t-s)^{\alpha-1} d s\right\}=0, \text { for all } t \in[0, T] .
$$

Therefore, $|w(t)|=0$ on $[-h, T]$. The proof is finished.

Remark 6. We prove the existence of solution for a general delay case, namely, $g\left(t, u_{t}\right)$ could be variable delay or distributed delay. In Section 4 , we take $g\left(t, u_{t}\right)=g(u(t-\tau(t)))$-i.e., the delay function $\tau(t) \in C\left(\mathbb{R}_{+} ;[0, h]\right)$, to study the dissipativity.

Remark 7. It is worth mentioning that only the existence result is proved under the condition that $\alpha \in\left(\frac{1}{2}, 1\right)$, which is due to the phase space $C([-h, 0] ; H)$. If $C([-h, 0] ; H)$ is replaced by some Sobolev space, such as $L^{2}((-h, 0) ; H)$. Then the existence of solution can be established for any $\alpha \in(0,1)$ and without additional conditions. 
Theorem 2. Suppose that $(g 1)-(g 3)$ hold true, then the solutions of system $(P)$ are continuous with respect of initial values-i.e.,

$$
\left\|u_{t}-v_{t}\right\|_{C_{H}}^{2} \leq c\|\phi-\varphi\|_{C_{H}}^{2} \exp \left\{c \int_{0}^{t}\left(\|v\|^{2}+1\right)(t-s)^{\alpha-1} d s\right\}, t \in[0, T]
$$

Proof. Let $u(t ; \phi), v(t ; \varphi)$ be the solutions of (1)-(4) with initial values, $\phi$ and $\varphi$, respectively. Set $w(t)=$ $u(t)-v(t)$ for $t>0$, and $w(t)=\phi(t)-\varphi(t)$ for $t \in[-h, 0]$. Then we have

$$
D_{t}^{\alpha} w-v \Delta w+B(u)-B(v)=g\left(t, u_{t}\right)-g\left(t, v_{t}\right), \quad t>0 .
$$

Multiplying above equation by $w(t)$, and integral over $\Omega$, we obtain

$$
\begin{aligned}
D_{t}^{\alpha}|w|^{2}+v\|w\|^{2} & =-(B(u)-B(v), w)+\left(g\left(t, u_{t}\right)-g\left(t, v_{t}\right), w\right) \\
& \leq c|w|^{2}\|v\|^{2}+2 L_{g}\left\|w_{t}\right\|_{C_{H}}^{2} \leq c|w|^{2}\|v\|^{2}+2 L_{g}\left(\|\phi-\varphi\|_{C_{H}}^{2}+\sup _{0 \leq t \leq T}|w|^{2}\right) \\
& \leq\left(c\|v\|^{2}+2 L_{g}\right) \sup _{0 \leq t \leq T}|w(t)|^{2}+2 L_{g}\|\phi-\varphi\|_{C_{H}}^{2} .
\end{aligned}
$$

Hence, we have

$$
\sup _{0 \leq t \leq T}|w(t)|^{2} \leq|w(0)|^{2}+\frac{2 L_{g} T^{\alpha}}{\Gamma(\alpha+1)}\|\phi-\varphi\|_{C_{H}}^{2}+\frac{1}{\Gamma(\alpha)} \int_{0}^{t}\left(c\|v\|^{2}+2 L_{g}\right)(t-s)^{\alpha-1} \sup _{0 \leq s \leq t}|w(s)|^{2} d s
$$

Again using the Bellman-Gronwall Lemma 3 and (21), we find that

$$
\left\|w_{t}\right\|_{\mathcal{C}_{H}}^{2} \leq c\|\phi-\varphi\|_{C_{H}}^{2} \exp \left\{c \int_{0}^{t}\left(\|v\|^{2}+1\right)(t-s)^{\alpha-1} d s\right\}, t \in[0, T] .
$$

The proof is complete.

\section{Dissipativity}

In this section, we derive some uniform estimates of solutions to problem $(P)$ by using Proposition 2. Besides, in this section, we assume that $g\left(t, u_{t}\right)=g(u(t-\tau(t)))$.

Definition 7. The system $(P)$ is said to be dissipative in $C_{H}$ if there exists a bounded set $B \subset C_{H}$, such that for any given bounded set $A \subset C_{H}$, there is a time $t^{*}=t^{*}(A)$, such that for any given initial function $\phi \in A$, for all $t \in[-h, 0]$, the values of the corresponding solution $u(t)$ of the problem $(P)$ are contained in $B$ for all $t \geq t^{*}$. The set $B$ is called an absorbing set of the system $(P)$.

We assume that

$$
\lambda_{1} v>\sqrt{2} L_{g} .
$$

Theorem 3. (Existence of absorbing sets in $C_{H}$ ) Assume that $(g 1)-(g 3),(17)$ and (32) hold. Then there exists $T>0$, such that for all $t \geq T$, the solution of problem $(P)$ satisfies

$$
\left\|u_{t}\right\|_{\mathcal{C}_{H}}^{2} \leq \frac{\lambda_{1} v f_{0}}{\left(\lambda_{1} v\right)^{2}-2 L_{g}^{2}}+1, \forall t \geq T
$$

where $f_{0}=v^{-1} \sup _{t \geq 0}\|f(t)\|_{*}^{2}$. 
Proof. Multiplying (1) by $u$, integrating over $\Omega$, we have

$$
\begin{aligned}
D_{t}^{\alpha}|u(t)|^{2}+v\|u(t)\|^{2} & \leq\|f(t)\|_{*}\|u(t)\|+|g(u(t-\tau(t))) \| u(t)| \\
& \leq \frac{\|f(t)\|_{*}^{2}}{v}+\frac{2 L_{g}^{2}}{\lambda_{1} v} \sup _{t-\tau(t) \leq s \leq t}|u(s)|^{2} .
\end{aligned}
$$

Then we obtain

$$
\begin{gathered}
D_{t}^{\alpha}|u(t)|^{2} \leq f_{0}-\lambda_{1} v|u(t)|^{2}+\frac{2 L_{g}^{2}}{\lambda_{1} v} \sup _{t-\tau(t) \leq s \leq t}|u(s)|^{2}, \quad t \in(0, T], \\
|u(t)|^{2}=|\phi(t)|^{2}, \quad t \in[-h, 0]
\end{gathered}
$$

where $f_{0}=v^{-1} \sup _{t \geq 0}\|f(t)\|_{*}^{2}$. Using Proposition 2, we find that

$$
|u(t)|^{2} \leq \frac{\lambda_{1} v f_{0}}{\left(\lambda_{1} v\right)^{2}-2 L_{g}^{2}}+M E_{\alpha}\left(\lambda^{*} t^{\alpha}\right)
$$

for all $t \geq \tau(t)$, where $M=\|\phi\|_{C_{H}}^{2}=\sup _{-h \leq t \leq 0}|\phi(t)|^{2}$, and the parameter $\lambda^{*}$ is defined by

$$
\lambda^{*}=\sup _{t-\tau(t) \geq 1}\left\{\lambda: \lambda-\left(-\lambda_{1} v\right)-\frac{2 L_{g}^{2}}{\lambda_{1} v} \frac{E_{\alpha}\left(\lambda(t-\tau(t))^{\alpha}\right)}{E_{\alpha}\left(\lambda t^{\alpha}\right)}=0\right\},
$$

is strictly negative, namely, there exists some positive constants $\epsilon_{0}$ satisfying $-\lambda_{1} v+\frac{2 L_{g}^{2}}{\lambda_{1} v}<-\epsilon_{0}$ such that $\lambda^{*} \in\left[-\lambda_{1} v+\frac{2 L_{g}^{2}}{\lambda_{1} v},-\epsilon_{0}\right]$, and the estimate in (9) holds for all $t$ such that $t \geq \tau(t)+1$. In other words, for $\lambda^{*} \in\left[-\lambda_{1} v+\frac{2 L_{g}^{2}}{\lambda_{1} v},-\epsilon_{0}\right]$, we have

$$
|u(t)|^{2} \leq \frac{\lambda_{1} v f_{0}}{\left(\lambda_{1} v\right)^{2}-2 L_{g}^{2}}+M E_{\alpha}\left(\lambda^{*} t^{\alpha}\right), \quad \forall t \geq \tau(t)+1 .
$$

For the case of $t<\tau(t)+1$, in order to analyze the dissipativity of problem $(P)$ in phase space $C_{H}$ by Proposition 3, we first need to consider the following fractional differential equation,

$$
\begin{aligned}
& D_{t}^{\alpha} w(t)+\lambda_{1} v w(t)=f_{0}+\frac{2 L_{g}^{2}}{\lambda_{1} v} w(t-\tau(t)), 0<t \leq h+1, \\
& w(t)=|\phi(t)|^{2}, \quad t \in[-h, 0]
\end{aligned}
$$

Then, by using the method of steps [28] (Theorem 1), we have that the initial value problem (34) has, on the interval $[0, k h]$, a unique solution that can be represented by $w(t)=w_{i h}(t)$, if $(i-1) h \leq t \leq i h$,

$$
w_{i h}(t)=\int_{0}^{t} E_{\alpha, \alpha}\left(-\lambda_{1} v(t-s)^{\alpha}\right) f_{i h}(s) d s+c_{i h} E_{\alpha}\left(-\lambda_{1} v t^{\alpha}\right), \quad t \in[(i-1) h, i h],
$$


where $c_{i h}$ is a constant, $i=1,2, \cdots, k$.

$$
f_{k h}(t):=\left\{\begin{array}{l}
\frac{2 L_{g}^{2}}{\lambda_{1} v} w_{0 h}(t-h)+f_{0}, \quad 0<t \leq h, \\
\frac{2 L_{g}^{2}}{\lambda_{1} v} w_{1 h}(t-h)+f_{0}, \quad h<t \leq 2 h, \\
\cdots \\
\frac{2 L_{g}^{2}}{\lambda_{1} v} w_{(k-1) h}(t-h)+f_{0}, \quad(k-1) h<t \leq k h,
\end{array}\right.
$$

is continuous and $w_{0 h}(t)=|\phi(t)|^{2} . k$ is a smallest integer such that $k h \geq h+1$. Therefore, we obtain that

$$
|w(t)| \leq \sum_{0 \leq i \leq k}\left|w_{i h}(t)\right| \leq C E_{\alpha}\left(-\lambda_{1} v t^{\alpha}\right), \forall 0 \leq t \leq \tau(t)+1
$$

Now, we estimate the solution of (1), for $t<\tau(t)+1$. By (33), we have

$$
\begin{gathered}
D_{t}^{\alpha}|u(t)|^{2}+\lambda_{1} v|u(t)|^{2} \leq \frac{\|f(t)\|_{*}^{2}}{v}+\frac{2 L_{g}^{2}}{\lambda_{1} v}|u(t-\tau(t))|, 0 \leq t<\tau(t)+1, \\
|u(t)|^{2}=|\phi(t)|^{2}, \quad t \in[-h, 0] .
\end{gathered}
$$

Then, by Proposition 3 and (34)-(36), we have

$$
|u(t)|^{2} \leq C E_{\alpha}\left(-\lambda_{1} v t^{\alpha}\right), \quad 0 \leq t<\tau(t)+1 .
$$

So, we find that

$$
|u(t)|^{2} \leq \frac{\lambda_{1} v f_{0}}{\left(\lambda_{1} v\right)^{2}-2 L_{g}^{2}}+M E_{\alpha}\left(\lambda^{*} t^{\alpha}\right)+C E_{\alpha}\left(-\lambda_{1} v t^{\alpha}\right), \text { for all } t \geq 0
$$

By the norm of $C_{H}$, we conclude that

$$
\left\|u_{t}\right\|_{C_{H}}^{2} \leq \frac{\lambda_{1} v f_{0}}{\left(\lambda_{1} v\right)^{2}-2 L_{g}^{2}}+M E_{\alpha}\left(\lambda^{*} t^{\alpha}\right)+C E_{\alpha}\left(-\lambda_{1} v t^{\alpha}\right), \text { for all } t \geq 0, \theta \in[-h, 0] .
$$

Since $\lambda^{*}$ and $-\lambda_{1} v$ are strictly negative, by the property of Mittag-Leffler function [2], we obtain

$$
\left\|u_{t}\right\|_{C_{H}}^{2} \leq \frac{\lambda_{1} v f_{0}}{\left(\lambda_{1} v\right)^{2}-2 L_{g}^{2}}+C \frac{C_{\alpha}}{t^{\alpha}}, \text { as } t \rightarrow+\infty
$$

where $C_{\alpha}>0$ is a constant independent of $t$. Therefore, there exists $T>0$ large enough, such that for all $t \geq T$, the solution of problem $(P)$ satisfies

$$
\left\|u_{t}\right\|_{C_{H}}^{2} \leq \frac{\lambda_{1} v f_{0}}{\left(\lambda_{1} v\right)^{2}-2 L_{g}^{2}}+1, t \geq T
$$

Denote by $B_{C_{H}}=B\left(0, \sqrt{\frac{\lambda_{1} v f_{0}}{\left(\lambda_{1} v\right)^{2}-2 L_{g}^{2}}+1}\right)$ the absorbing set in phase space $C_{H}$, which implies that system $(P)$ is dissipative. The proof is complete. 


\section{Discussion}

In this work, we prove the existence and uniqueness of solution for fractional Navier-Stokes equations with variable delays for $\alpha \in\left(\frac{1}{2}, 1\right)$, and we show that this system is dissipative in the phase space $C_{H}$, namely, there exists a global absorbing set in $C_{H}$. Different from the classic Navier-Stokes equations with variable delays [22-24], in which the existence of pullback absorbing set and pullback attractors were established. Here, we obtained the forward absorbing set, which is more meaningful from the view of applications. Besides, the existence of global attracting set as well as the existence of solution for $\alpha \in(0,1)$ in phase space $C_{H}$ are still open problems. These will be considered in the future.

Author Contributions: Conceptualization, L.F.L. and J.J.N.; methodology, L.F.L. and J.J.N.; writing-original draft preparation, L.F.L.; writing-review and editing, J.J.N. All authors have read and agreed to the published version of the manuscript.

Funding: The work of Lin F. Liu has been partially supported by NSF of China (Nos. 11901448, 11871022 and 11671142) as well as by China Postdoctoral Science Foundation Grant (Nos. 2018M643610). The work of Juan J. Nieto has been partially supported by the Agencia Estatal de Investigación (AEI) of Spain, co-financed by the European Fund for Regional Development (FEDER) corresponding to the 2014-2020 multiyear financial framework, project MTM2016-75140-P, Xunta de Galicia under grant ED431C 2019/02; by Instituto de Salud Carlos III (Spain), grant COV20/00617.

Conflicts of Interest: The authors declare no conflict of interest.

\section{References}

1. Piero, G.; Deseri, L. On the concepts of state and free energy in linear viscoelasticity. Arch. Rat. Mech. Anal. 1997, 138, 1-35. [CrossRef]

2. Kilbas, A.A.; Srivastava, H.M.; Trujillo, J.J. Theory and Applications of Fractional Differential Equations; Elsevier Science B.V.: Amsterdam, The Netherlands, 2006.

3. Podlubny, I. Fractional Differential Equations. In An Introduction to Fractional Derivatives, Fractional Differential Equations, To Methods of Their Solution and Some of Their Applications; Academic Press Inc.: San Diego, CA, USA, 1999.

4. Mainardi, F.; Paradisi, P.; Gorenflo, R. Probability distributions generated by fractional diffusion equations. arXiv 2007, arXiv:0704.0320.

5. Diethelm, K. The Analysis of Fractional Differential Equations: An Application-Oriented Exposition Using Differential Operators of Caputo Type; Springer: Berlin, Germany, 2010.

6. Caputo, M. Linear models of dissipation whose $Q$ is almost frequency independent-II. Geophys. J. Intern. 1967, 13, 529-539. [CrossRef]

7. Allen, M.; Caffarelli, L.; Vasseur, A. A parabolic problem with a fractional-time derivative. Arch. Ration. Mech. Anal. 2016, 221, 603-630. [CrossRef]

8. Li, L.; Liu, J. A Generalized Definition of Caputo Derivatives and Its Application to Fractional ODEs. SIAM J. Math. Anal. 2018, 50, 2867-2900. [CrossRef]

9. Chaurasia, V.; Kumar, D. Solution of the Time-Fractional Navier-Stokes Equation. Gen. Math. Notes 2011, 4, 49-59.

10. Zhou, Y.; Peng, L. On the time-fractional Navier-Stokes equations. Comp. Math. Appl. 2017, 73, 874-891. [CrossRef]

11. de Carvalho-Neto, P.M.; Planas, G. Mild solutions to the time fractional Navier-Stokes equations in $R^{n}$. J. Differ. Equ. 2015, 259, 2948-2980. [CrossRef]

12. Gorenflo, R.; Luchko, Y.; Yamamoto, M. Operator theoretic approach to the caputo derivative and the fractional diffusion equations. arXiv 2014, arXiv:1411.7289.

13. Chen, F.; Nieto, J.J.; Zhou, Y. Global attractivity for nonlinear fractional differential equations. Nonlinear Anal. RWA 2012, 13, 287-298. [CrossRef]

14. Harikrishnan, S.; Prakash, P.; Nieto, J.J. Forced oscillation of solutions of a nonlinear fractional partial differential equation. Appl. Math. Comput. 2015, 254, 14-19. [CrossRef] 
15. Ye, H.; Gao, J.; Ding, Y. A generalized Gronwall inequality and its application to a fractional differential equation. J. Math. Anal. Appl. 2007, 328, 1075-1081. [CrossRef]

16. Ye, H.; Gao, J. Henry-Gronwall type retarded integral inequalities and their applications to fractional differential equations with delay. Appl. Math. Comp. 2011, 218, 4152-4160. [CrossRef]

17. Li, L.; Liu, J. Some compactness criteria for weak solutions of time fractional PDEs. SIAM J. Math. Anal. 2018, 50, 3963-3995. [CrossRef]

18. Wen, L.; Yu, W.; Wang, W. Generalized Halanay inequalities for dissipativity of Volterra functional differential equations. J. Math. Anal. Appl. 2008, 347, 169-178. [CrossRef]

19. Wang, D.; Zou, J. Dissipativity and contractivity analysis for fractional functional differential equations and their numerical approximations. SIAM J. Numer. Anal. 2019, 57, 1445-1470. [CrossRef]

20. Wang, H.; Yu, Y.; Wen, G.; Zhang, S.; Yu, J. Global stability analysis of fractional-order Hopfield neural networks with time delay. Neurocomputing 2015, 154, 15-23. [CrossRef]

21. Liu, L.F.; Caraballo, T.; Marín-Rubio, P. Stability results for 2D Navier-Stokes equations with unbounded delay. J. Differ. Equ. 2018, 265, 5685-5708. [CrossRef]

22. Marín-Rubio, P.; Real, J. Pullback attractors for 2D-Navier-Stokes equations with delays in continuous and sub-linear operators. Discrete Cont. Dyn. Syst. A 2012, 26, 989-1006. [CrossRef]

23. Marín-Rubio, P.; Real, J.; Valero, J. Pullback attractors for a two-dimensional Navier-Stokes model in an infinite delay case. Nonlinear Anal. 2011, 74, 2012-2030. [CrossRef]

24. Caraballo, T.; Real, J. Attractors for 2D-Navier-Stokes models with delays. J. Differ. Equ. 2004, 205, 271-297. [CrossRef]

25. Gel'fand, I.M.; Shilov, G.E. Generalized Functions; Academic Press: New York, NY, USA, 1964; Volume 1.

26. Alikhanov, A. A priori estimates for solutions of boundary vale problems for fractional-order equations. Differ. Equ. 2010, 46, 660-666. [CrossRef]

27. Simon, J. Compact sets in the space $L^{p}(0, T ; B)$. Ann. Mat. Pure Appl. 1987, 146, 65-96. [CrossRef]

28. Morgadoa, M.L.; Ford, N.J.; Lima, P.M. Analysis and numerical methods for fractional differential equations with delay. J. Comput. Appl. Math. 2013, 252, 159-168. [CrossRef]

29. Desoer, C.A.; Vidyasagar, M. Feedback Systems: Input-Output Properties; Academic Press: New York, NY, USA, 1975.

30. Lakshmikantham, V. Theory of fractional functional differential equations. Nonlinear Anal. 2008, 69, 3337-3343. [CrossRef]

Publisher's Note: MDPI stays neutral with regard to jurisdictional claims in published maps and institutional affiliations. 